\title{
DER DOKUMENTARISCHE GESTUS. EINE SPURENSUCHE IN POPULÄRER MUSIK UND KULTUR IN DER BRD DER 1970ER JAHRE
}

\author{
Barbara Hornberger
}

\section{Rauch-Haus-Song}

Am 8. Dezember 1971 wird nach einem Teach-In anlässlich des Todes von Georg von Rauch in der TU Berlin das leerstehende Martha-Maria-Haus, ein ehemaliges Schwesternwohnheim auf dem Gelände des Bethanien-Krankenhauses, besetzt. Die Band Ton Steine Scherben, die bei dem Teach-In gespielt hat, ist an der Aktion beteiligt. Der Polizei gelingt es nicht, die Besetzung zu verhindern, Verhandlungen führen dazu, dass das Haus zunächst nicht geräumt wird. ${ }^{1}$ Es wird von den Besetzern nach dem »umherschweifenden Haschrebellen $\ll^{2}$ Georg von Rauch benannt, der wenige Tage vor dem besagten Teach-In nach einem Schusswechsel mit der Polizei gestorben war.

Am 19. April 1972 um 4:15 Uhr wird in dem besetzten Haus eine Großrazzia durchgeführt. Die Polizei findet leere Weinflaschen, Batterien und einen Wecker, ein kaputtes Wasserrohr und einiges an Hobbywerkzeug und glaubt damit zu beweisen, dass hier Sprengstoffanschläge geplant werden. Aufgrund dieser Beweise, die die Besetzer als konstruiert empfinden, soll das sogenannte Georg-von-Rauch-Haus geräumt werden.

Über diese Ereignisse schreibt der beteiligte Rio Reiser im Jahr 1972 einen Song für seine Band Ton Steine Scherben: den »Rauch-Haus-Song «.

1 Laut der Homepage des Georg-von-Rauch-Hauses (http://www.rauchhaus1971. de; Stand vom 23.7.2012) wurde nach einem Monat ein Nutzungsvertrag vereinbart. Im Jahr 1973 schließlich beschließt der Berliner Senat, im BethanienHauptgebäude das »Künstlerhaus Bethanien« einzurichten.

2 Von Rauch gehört einer militant linken Gruppe an, die sich »Zentralrat der umherschweifenden Haschrebellen « nannte; sie wird als eine Vorstufe zur Bewegung 2. Juni angesehen. 
Ton Steine Scherben: »Rauch-Haus-Song« (1972)

Der Mariannenplatz war blau, soviel Bullen waren da, und Mensch Meier musste heulen, das war wohl das Tränengas. Und er fragt irgendeinen: »Sag mal, ist hier heut 'n Fest?« "Sowas ähnliches«, sacht einer, »das Bethanien wird besetzt.« »Wird auch Zeit«, sachte Mensch Meier, »stand ja lange genug leer. Ach, wie schön wär doch das Leben, gäb es keine Pollis mehr.« Doch der Einsatzleiter brüllte: »Räumt den Mariannenplatz, damit meine Knüppelgarde genug Platz zum Knüppeln hat!«

Doch die Leute im besetzen Haus

riefen: »Ihr kriegt uns hier nicht raus!

Das ist unser Haus, schmeißt doch endlich

Schmidt und Press und Mosch aus Kreuzberg raus.«

Der Senator war stinksauer, die CDU war schwer empört, dass die Typen sich jetzt nehmen, was ihnen sowieso gehört.

Aber um der Welt zu zeigen, wie großzügig sie sind, sachten sie: »Wir räumen später, lassen sie erstmal drin!« Und vier Monate später stand in Springer's heißem Blatt, dass das Georg-von-Rauch-Haus eine Bombenwerkstatt hat. Und die deutlichen Beweise sind zehn leere Flaschen Wein und zehn leere Flaschen können schnell zehn Mollies sein.

Doch die Leute im Rauch-Haus

riefen: »Ihr kriegt uns hier nicht raus!

Das ist unser Haus, schmeißt doch endlich

Schmidt und Press und Mosch aus Kreuzberg raus.«

Letzten Montag traf Mensch Meier in der U-Bahn seinen Sohn.

Der sagte: »Die woll'n das Rauch-Haus räumen, ich muss wohl wieder zu Hause wohnen.« »Is ja irre«, sagt Mensch Meier, »sind wa wieder einer mehr in uns'rer Zweiraum Zimmer Luxuswohnung, und das Bethanien steht wieder leer.

Sag mir eins, ha'm die da oben Stroh oder Scheiße in ihrem Kopf?

Die wohnen in den schärfsten Villen, unsereins im letzten Loch.

Wenn die das Rauch-Haus wirklich räumen, bin ich aber mit dabei und hau den ersten Bullen, die da auftauchen, ihre Köppe ein.«

Und ich schrei's laut:

»Ihr kriegt uns hier nicht raus!

Das ist unser Haus, schmeißt doch endlich

Schmidt und Press und Mosch aus Kreuzberg raus.« 
Reiser gießt die Ereignisse um das Rauch-Haus in die Form eines Popsongs. Dabei verweist er direkt auf die Geschehnisse - und auf die Perspektive, die die Band dabei einnimmt. »Der Mariannenplatz « als Adresse des so genannten Rauch-Hauses markiert den Ort des Geschehens, der wenig später auch als »Bethanien « ausgewiesen und so mit einem »bürgerlichen « Namen benannt und für Nicht-Beteiligte identifizierbar gemacht wird. Seine blaue Farbe verdankt der Mariannenplatz der anwesenden Polizei - denn bis Mitte der 1970er Jahre sind die Fahrzeuge und Uniformen der Berliner Polizei blau. So wie der Ort sind auch die im Song genannten Namen Klarnamen: Schmidt, Mosch und Press sind Bauunternehmer, die das Neue Kreuzberger Zentrum am U-Bahnhof Kottbusser Tor errichteten und deren Bau- und Sanierungs-Pläne auch das Bethanien-Gelände tangierten. ${ }^{3}$ In der zweiten Strophe nimmt der Song mit »Springers heißes Blatt« auf die Auseinandersetzungen zwischen der Springer-Presse und der linken Szene Bezug: In der zum Springer-Verlag gehörenden B.Z. etwa war am Tag nach der Razzia die durch Alliterationen dekorierte Schlagzeile zu lesen: »Bastelten BethanienBesetzer die Bomben?« (Berndt 1972). Von der literarischen StellvertreterFigur »Mensch Meier « wird in der ersten und dritten Strophe auf den Leerstand der Bethanien-Gebäude und damit auf das Interesse der Bevölkerung an dem Gebäude verwiesen: Den ursprünglich geplanten Abriss der Gebäude nach der Stilllegung hatten Bürgerinitiativen verhindert.

Die Ereignisse und die Razzia im Rauch-Haus werden von Reiser verdichtet und in eine Erzählung für und über »Mensch Meier « verwandelt. Sie werden dabei nicht metaphorisiert, verfremdet oder verallgemeinert. Der

3 Bei der Aneignung des eingeplanten Geländes gingen die Verantwortlichen laut Spiegel nicht zimperlich vor: »Am Kottbusser Tor wollte Immobilien-Makler Günter Schmidt in halbkreisförmigem Bogen Gewerbe- und Wohnbauten für 80 Millionen Mark hochziehen. Doch auf dem Gelände standen noch Häuser, aus denen die Mieter nicht weichen mochten. Bauherr Schmidt sah sein >Neues Kreuzberger Zentrum < in Gefahr und beschloß, die >Entmietung der Altbauten zu forcieren: Ohne Vorwarnung ließ er Türen und Fenster des noch teilbewohnten Hauses Dresdener Straße 131 herausbrechen und auf den Hof werfen. Begründung: 'Wir wollen nicht, daß sich Gastarbeiter und anderes Gesindel einnisten « (Anon. 1973: 38/41).

»Mit der Axt brachen Abrißarbeiter den Widerstand der letzten Mieter des Hauses Dresdener Straße 131 im West-Berliner Bezirk Kreuzberg. Der Terrorangriff geschah 1971 - als die Abschreibungsgesellschaft >Neues Kreuzberger Zentrum KG Günter Schmidt Beteiligungen GmbH \& Co.< es nicht erwarten konnte, auf den frei gemachten Grundstücken im alten Postzustellbezirk SO 36 eine Geldgrube auszuheben« (Anon. 1977: 216). Dass sich sieben Jahre später ein Punk-Club SO 36 nennt, kann aus diesem Grund in einen Zusammenhang insbesondere mit Ton Steine Scherben und dem »Rauch-Haus-Song « gebracht werden. 
Song bleibt konkret, auch in der Musik: Das unaufwändige Arrangement vermittelt Nähe, das eher abgenutzt klingende Klavier und die SchrammelGitarre Sit-In-Feeling. Die einfache und eingängige Melodie eignet sich vor allem im Refrain bestens zum Mitsingen oder auch Mitgrölen - konsequenterweise wird der Gesang im Refrain durch einen Chor verstärkt, so dass der Hörer ein »Fast-Dabei«-Erlebnis hat. Auch Reisers Gesangstil stellt Unmittelbarkeit her: Er liegt zwischen Singen und Sprechen, ohne hochdeutsche Überformung, unter Einbeziehung von Szene-Jargon - der Unterschied zu Interviews mit Reiser liegt eigentlich nur in der Reimform und der tonalen Amplitude. Auf vielfältige Weise wird so das Hausbesetzungs-Milieu inhaltlich und musikalisch eingelöst und verewigt.

Mit dem »Rauch-Haus-Song « schreibt Rio Reiser mehr als nur einen demotauglichen Song. Er stellt das Ereignis »Bethanien-Besetzung « auch nicht einfach dar, er dokumentiert es vielmehr. Dadurch verankert er Geschichte im kulturellen Gedächtnis - zunächst für die eigene Szene, dann aber auch für die gesellschaftliche Öffentlichkeit.

\section{Willy}

Fünf Jahre nach dem »Rauch-Haus-Song « und $580 \mathrm{~km}$ südlich sitzt in München ein junger Liedermacher am Klavier und besingt das Leben und den Tod seines Freundes »Willy«. Der Song in bayrischer Mundart ist eine Trauerrede in Du-Form, der Verstorbene wird beweint, für seine Ehrlichkeit gefeiert, für seine Sturheit verdammt. Mit der Blues-Ballade lässt der Sänger Konstantin Wecker Willys Leben, besonders die letzten Stunden dieses Lebens, Revue passieren. Dabei wird das individuelle Leben des Betrauerten verflochten mit der jüngeren Geschichte der BRD. Aus Willys - und Weckers - Blickwinkel endet die politische Rebellion der 68er als Mode-Erscheinung, sind die hohen Ziele durch Mitläufer diskreditiert. Willy verzweifelt am gesellschaftlichen Stillstand, sucht das »Echte « und wird am Ende in einer echten Kneipenschlägerei von einem Rechtsradikalen erschlagen. Die große Geschichte der Revolution und ihres Versickerns wird erzählt in der Lebensgeschichte eines kleines Revoluzzers.

Die Geschichte des aufrechten Weltverbesserers Willy erhält ihre exemplifizierende Kraft durch den Einsatz von Verfahren, die ebenfalls dokumentarisch genannt werden können: Zum einen wird aus der Perspektive des zurückgebliebenen Weggefährten das Leben und der Tod eines Rebellen geschildert, zum anderen der Niedergang der 68er-Bewegung. Allerdings sind die eingesetzten Mittel andere als bei Ton Steine Scherben. Ein zentra- 
les Element des Songs ist der Dialekt, die Strophen sind lang und weitschweifend erzählerisch, gesprochen in nahezu unbereinigtem Bayrisch, nur die kurzen Refrainzeilen sind auch für Nicht-Mundartler zu verstehen. Dadurch stellt sich einerseits Distanz her, denn anders als bei Ton Steine Scherben ist dieser Text keineswegs leicht zugänglich. Andererseits zeigt dieser Sprachduktus den Sänger als »ehrlich« und unverstellt, macht den Song direkt und unmittelbar. Damit wird die im Text hergestellte Behauptung des »gestern« - also des »gerade erst « beglaubigt. Nicht-bayrische Zeilen sind als Zitate identifizierbar: Die Anarcho-Parole »Bürger lasst das Glotzen sein « - wird von den Zuhörern als Original-Quelle wiedererkannt. Betrachtet man eine Aufführung des Songs, so kommt diese schlicht, nur mit Klavier, und zugleich pathetisch daher. Soweit es das Instrument überhaupt zulässt, ist Wecker körperlich involviert, womit die Geschichte emotional noch mehr aufgeladen wird: Der Oberkörper schwankt, es hält ihn kaum auf dem Sitz, der Griff in die Tasten ist kraftvoll, auf dem Gesicht steht der Schweiß, die geschlossenen Augen signalisierten Innerlichkeit und Schmerz: Hier wird Arbeit geleistet. Trauerarbeit.

\section{Der dokumentarische Gestus}

Konstantin Wecker transportiert die Botschaft seines Songs in seinem Auftritt auch über den Körper, genauer: über die Mimik und - soweit die Gesetze des Klavierspiels das zulassen - über die Gestik. Seine politische Haltung vermittelt sich über die körperliche Haltung. Bei Wecker wird augenfällig, was Brecht meinte, als er den Begriff des Gestus für seine theatertheoretischen Überlegungen in Anspruch nahm: Die Geste wird zum Gestus, wenn durch sie nicht nur eine Handlung vollzogen, sondern eine gesellschaftliche Haltung demonstriert wird. Der Gestus verweist in diesem Verständnis nicht mehr nur auf die Sprache, die er begleitet oder sogar substituiert, auch nicht mehr (nur) auf den ausführenden Körper ${ }^{4}$, sondern auf die »gesellschaftlichen Beziehungen [...], in denen die Menschen einer bestimmten Epoche zueinander stehen « (Brecht 1963: 163). Brecht verdeutlicht diesen Aspekt mit dem Begriff des sozialen oder gesellschaftlichen Gestus. Wesentlich ist dabei, dass der Gestus des Zeigens der Geste das Expressive nimmt und auf das Gesellschaftliche verweist. Dieses Zeigen verleiht dem Gestus-Begriff auch außerhalb des theatralen Kontexts Beschreibungskraft. Zwar leidet außerhalb dieses theatralen Rahmens die Präzision

4 »Auch Sprache oder Musik können gestischen Charakter haben und einen Gestus zur Darstellung bringen« (Kuba 2005: 134). 
des Begriffs spürbar, eine kulturwissenschaftliche Begriffsdefinition fehlt bisher ebenso wie eine stringente Anwendungstradition. ${ }^{5}$ Dennoch kann mit dem Begriff des Gestus die Qualität einer Kunst, eines Formats, eines Genres, eines einzelnen Werks oder einer Handlung beschrieben werden, die nicht Stil zu nennen ist - der den künstlerischen Ausdruck einer Epoche oder eines Künstlers meint - und nicht Handschrift - die auf die individuelle Person des Künstlers verweist - , die aber eben auch nicht an die Geste selbst, also den Körper, gebunden ist - wie bei Flusser (1993). Die wesentliche Eigenschaft, die mit dem Begriff Gestus beschrieben werden kann und soll, ist die eines Zeigens, genauer: der Demonstration. Was im Einzelnen gezeigt werden soll, muss jeweils benannt werden.

In den beiden oben beschriebenen Beispielen - und den noch folgenden - bezieht sich das Zeigen auf das Dokumentarische. Allerdings, so könnte man einwenden, ist Musik kaum eine Kunstform, die sich für Dokumentation besonders eignet. Allein ihre tonal und rhythmisch-metrisch determinierte Gestalt bringt eine Form von Verfremdung und Überformung mit sich, die die Mimesis stört, zwangsläufig Brüche produziert, die aus dem Einen - dem Ereignis - stets etwas Anderes - Musik - macht. Zwar ist auch die Dokumentation in print- oder audiovisuellen Medien eine gestaltete, also neue, Form. Dort aber wird die Nähe zum Ereignis regelmäßig durch die Einarbeitung von Originalmaterial und Quellen belegt, durch eine Mischung aus Interviews, Zeitzeugen und Fotos, mit der ein nichtfiktionaler, glaubwür-

5 Zwar wird implizit immer auf das Zeigen oder Zur-Schau-Stellen verwiesen, davon abgesehen ist der Begriff außerhalb der Brechtschen Theorie deutlich unterdeterminiert. Häufig und keineswegs nur im Alltagsgebrauch wird er mit einem Adjektiv verwendet - romantischer Gestus, neo-barocker Gestus, illustrierender Gestus, sinfonischer Gestus - oder mit einem nachgestellten Genitiv - Gestus der Trauer, Gestus der Revolte, Gestus der Freiheit -, ohne dass der Begriff des Gestus selbst dabei geklärt wäre. Gerade im Zusammenhang mit Musik und Film wird er regelmäßig benutzt, doch selbst dann, wenn er explizit, z.B. im Titel, eingeführt wird, findet eine Begriffsklärung kaum oder gar nicht statt (vgl. z. B. Berg 1987 oder Jacob 2009). Wenn überhaupt zum Begriff erklärend Stellung bezogen wird, dann erfolgt die Herleitung und Legitimation meist mehr (Burde 1980) oder weniger (Koch 2010) deutlich über Brecht. Burde stellt dabei immerhin die Frage, wie das Soziale in die Musik kommt: "Fragwürdig bleibt dennoch, wie die musikalische Gestik zur Konkretion einer gesellschaftlichen Haltung gerinnen kann, zum Gestus zu werden vermag « (Burde 1980: 531). In seinen daran anschließenden Analysebeispielen ist es das Miteinander von Musik und Text, das eine gesellschaftliche Gesamthaltung erkennen lässt. $\mathrm{Da}$ ein rein musikalisches Beispiel fehlt, liegt hier die Vermutung nahe, dass erstens die Musik dem Text folgt und zweitens der Text den Gestus-Charakter im Brechtschen Sinne besitzt und nicht die Musik.

Die Begriffe Geste, Gestik und Gestus werden überdies bei Koch wie auch bei anderen häufig synonym gebraucht. Eine allgemeine kulturwissenschaftlichphilosophische Klärung des Begriffs steht offenbar noch aus. 
diger Blick auf die Realität hergestellt wird. Musik dagegen bleibt auch dort, wo sie sich mehr oder weniger auf reale Ereignisse bezieht, in erster Linie Musik und wird nicht etwa zum journalistisch aufbereiteten Bericht. Mit Musik wird demzufolge Dokumentation als Verfahren vor allem dort in Verbindung gebracht, wo Musik bzw. das Musizieren dargestellt wird - DVDs von Rockkonzerten, Festival-Filme, Backstage-Aufnahmen, Making-Ofs zeigen uns, was passiert, wenn Musik gemacht wird. Die Musik dokumentiert hier sich selbst und wird nicht zur Dokumentation von etwas anderem.

Im »Rauch-Haus-Song « und Weckers »Willy« aber wird erkennbar etwas Außermusikalisches dokumentiert. Während der »Rauch-Haus-Song « den Aufbruch der Hausbesetzerszene markiert, ist Weckers »Willy« bereits ein Abgesang auf die 68er-Bewegung, die Ende der 1970er Jahre weitgehend zersplittert und zerstritten ist. Beide Songs beziehen sich auf die linke Revolte - und natürlich macht dies sie auf gewisse Weise zu Dokumenten - so wie jedes kulturelle Erzeugnis immer auch Dokument seiner Zeit ist. Um diese banale Erkenntnis geht es mir aber nicht, wenn ich im Zusammenhang mit diesen Songs von einem dokumentarischen Gestus spreche. Mit dem Begriff des Gestus wird gerade deutlich gemacht, dass es sich nicht um den zwangsläufigen zeithistorischen Gehalt eines Artefakts handelt, sondern um eine bewusste formalästhetische Strategie, nicht um Authentizität, sondern um Darstellung. Das Dokumentarische ist hier kein Ausweis von echtem »SoSein«, es ist auch keine Zuschreibung der Rezeption, sondern es werden absichtlich Mittel eingesetzt, die dokumentarisch wirken - auch dort, wo im fiktionalen Kontext gar nichts wirklich dokumentiert wird.

Der dokumentarische Gestus erfasst den intentionalen Einsatz ästhetischer Mittel und Formen, die wir als dokumentarisch identifizieren: Zeitungsausschnitte zum Beispiel, das Zitat, das als Quelle deutlich ausgewiesen wird, öffentliche Dokumente, der Bezug zur Realität, der durch Daten, Ortsnennungen und Klarnamen hergestellt wird. Es geht um den Einsatz jener Ästhetik, mit der in den Dokumentationen die »Echtheit « des Materials beglaubigt und damit die Wahrhaftigkeit der getroffenen Aussage belegt wird. Dieser Gestus ist im »Rauch-Haus-Song « und in »Willy« nachweisbar. Die Verweise auf Realität sind klar und unverkennbar, die Texte arbeiten mit Zitaten und Klarnamen und die Präsentationsformen zeigen - auf unterschiedliche Art, nämlich durch die biografische Involviertheit der Band Ton Steine Scherben in die erzählte Geschichte und durch die mit dem Bayrischen und der emotionalen Vortragsweise zusätzlich beglaubigte IchErzählung bei Wecker - die Künstler als Zeugen der dokumentierten Ereignisse. Damit bleiben allerdings beide Songs nah an den Authentifizierungsstrategien, die mit dokumentarischen Verfahren häufig verknüpft werden 
und speziell in diesen politisch aufgeladenen Songs zur Beglaubigung wesentlich sind.

\section{Maschinenland}

Von derartiger Leidenschaft und persönlicher Involviertheit scheint der Song »Maschinenland« von der Hamburger NDW-Band Abwärts (1980) weit entfernt zu sein. Der Song feiert nichts, fordert nichts und betrauert nichts, er kennt keine Subjekte und keine Position. Dennoch dokumentiert der Song etwas und zwar nüchtern und leidenschaftslos wie eine Kamera. Damit entspricht er einer Idee des Punk, nämlich der Forderung nach einem unmittelbaren Ausdruck der eigenen Welterfahrung. Der Transfer dieser Idee führt Anfang der 1980er Jahre in Deutschland nicht nur zu einem Boom deutscher Texte, sondern ist verbunden mit dem Anspruch, Realität abzubilden. Es geht in den NDW-Songs dieser Phase nicht um utopische Weltentwürfe, um Traumbilder oder Fantasiewelten, es geht auch selten um Liebe oder Schmerz. Sie suchen stattdessen einen neuen, ungeschminkten Zugriff auf die Gegenwart.

Abwärts: »Maschinenland « (1980)

linke seite supermarkt

rechte seite abenteuerspielplatz

in der mitte autobahn

lalalala

maschinenland maschinenland

wann bist du denn wohl abgebrannt

Der Text des Songs ist extrem reduziert: $\mathrm{Er}$ ist nur sechs Zeilen lang. $\mathrm{Er}$ kommt ohne Strophen oder Refrain aus, ohne durchlaufendes Metrum, ohne Verse und fast ohne Reim. Bis auf die letzte Zeile hat der Song weder Verben noch Adjektive. Die Zeilen wirken darum wie Parolen oder Schlagzeilen. Bei aller Knappheit besitzt der Song eine neutral-beschreibende Qualität. Mit den ersten drei Zeilen wird eine unwirtliche, menschenfeindliche Umwelt skizziert. Die nüchterne Sachlichkeit des Textes entspricht dabei völlig dem Charakter der beschriebenen Umgebung. Supermarkt, Abenteuerspielplatz, Autobahn - mehr gibt es zu diesem Ort nicht zu sagen. Und gerade weil die Beschreibung so unspezifisch ist, hat sie einen hohen Wiedererkennungswert: Solche Orte urbaner Ödnis müssen nicht detailliert beschrieben werden, die kennt ohnehin nahezu jeder. 
Der Charakter des Songs ist geprägt durch seinen besonderen Sound: eine Mischung aus Standard-Instrumentarium und Geräuschen, die sowohl als Effekt als auch rhythmisch-instrumental eingesetzt werden. Eine anlaufende Bohrmaschine, eine Säge, eine Flex, etwas Rasselndes - eine ganze Kaskade von Handwerksgeräuschen, eine regelrechte Lärmkulisse wird eingesetzt, um das »Maschinenland « zu illustrieren. Damit wird der Titel des Songs von Beginn an lautmalerisch umgesetzt. Darunter liegt ein gleichlaufender, durchgehender Beat. Dieser gleichmäßig durchlaufende Rhythmus produziert eine Atmosphäre maschineller Monotonie, die den GeräuschEinsatz noch verstärkt. Der dokumentarische Gestus reicht hier bis ins Soundmaterial: Mit den Geräuschen wird die im Text entworfene Skizze angereichert. Das Maschinenland wird musikalisch illustriert. Sogar das »lalala«, eigentlich eine Zeile, die Fröhlichkeit und Unbeschwertheit signalisiert, wird monoton, beinahe roboterhaft vorgetragen. So wird das Kindliche, das auch im Zitat der letzten Zeile steckt (»Maikäfer, flieg«), konsequent unterlaufen und damit die Lebensfeindlichkeit dieser Umgebung, die gerade - Abenteuerspielplatz hin oder her - nicht kindgerecht ist, noch hervorgehoben. Die enge Verbindung von Musik und Text schafft eine hohe Verdichtung. Der Anspruch, Wirklichkeit zu formulieren, wird hier dokumentarisch erfüllt: Keine Ich-Perspektive relativiert die Beschreibung; im telegrammartigen Headline-Stil wird nur das Sichtbare aufgezählt. Der Verweis auf das Kinderlied bricht dies nicht auf, sondern verstärkt den Eindruck: Aus dieser Ödnis gibt es keinen Ausweg.

Mit Songs wie »Maschinenland« findet die NDW eine Form des dokumentarischen Gestus, die nicht in erster Linie auf den Gehalt der Erzählung rekurriert, sondern in der Formensprache selbst verankert ist. Hier wird besonders deutlich, dass es nicht um Authentifizierung, nicht um Beglaubigung geht, sondern dass der Gestus selbst zum zentralen Prinzip wird, zum künstlerischen Verfahren, das sich scheinbar ideologie- und milieufrei nur dem Abbilden widmet. Diese Strategie ist zumindest teilweise auch in NDW-Songs wie »Rank Xerox« (Hans-A-Plast, 1979) oder »Der lange Weg nach Derendorf « (Mittagspause, 1979) erkennbar, die mit dem direkten Aufgreifen der historischen Ereignisse der 1970er Jahre inhaltlich eine größere inhaltliche Verwandtschaft zum »Rauch-Haus-Song « haben, aber eine viel stärkere Form der Verrätselung betreiben und damit die breite Identifizierung erschweren: Die Zeile »Ein Kreuz für jeden, den's erwischt« (»Rank Xerox«) muss vom Hörer auf die kopierten Fahndungsplakate erst einmal bezogen werden; das Wissen um die Erschießung Willy Peter Stolls im Düsseldorfer Stadtteil Derendorf ist das Spezialwissen einer politisch engagierten Szene. 


\section{Intertextuelle Spurensuche}

Bei aller Transformation, die in den ästhetischen Bedingungen der Form »Popsong « steckt, bleibt in den drei hier vorgestellten Songs der dokumentarische Gestus gebunden an einen Verweis auf Realität. Diese spezifische inhaltliche und ästhetische Art von Bezugnahme ist in der Epoche der 1970er Jahren in zahlreichen Artefakten zu finden, und zwar insbesondere dort, wo es sich um fiktionale Erzählungen handelt. Es ist sicher nicht weiter erstaunlich, dass ein Buch wie Christiane F. - Wir Kinder vom Bahnhof Zoo (1978) sich selbst durch Zeitzeugenberichte und Fotos und ein journalistisches Editorial als narrativ überformte Reportage zu erkennen gibt das Buch ist aus einer Reportage entstanden und zeigt auch aus Gründen der Glaubwürdigkeit die formensprachlichen Spuren dieser Entstehungsgeschichte. Der daraus entstandene Film hingegen zeigt sich eher als durcherzählter Spielfilm, bei dem das Wissen um die dokumentarische Form der Vorlage den Realitätsbezug ebenso herstellt wie der Einsatz von Laiendarstellern.

Überraschender ist die Art und Weise, wie Milos Forman in seinem Film Hair den Realitätsbezug herstellt. Zwar greift das Musical die Hippiebewegung der späten 1960er Jahre auf und zeigt sich damit klar auf Zeitgeschichte bezogen - doch weder das Bühnengenre Musical noch der Musicalfilm werden gemeinhin mit naturalistischen und realistischen Erzählungen assoziiert. Das in den Szenen (und Szenerien) entstehende Singen und Tanzen bedeutet stets eine starke Verfremdung, eine Künstlichkeit, die von jeder Form dokumentarischer oder realitätsnaher Darstellung weit entfernt scheint. Formans Film, der aus der Perspektive von 1979 nicht nur Abbildung, sondern auch Rückschau auf diese Bewegung ist, lässt seinen Film mit einem dokumentarischen Bild enden: Die große Demonstration, die in bewegten und bunten Bildern die bewegten und bunten Demonstranten zeigt, gefriert in einem zeitungsähnlichen Schwarz-Weiß-Bild, wie man es von den Pressefotos der Zeit kennt. Die durchaus desillusionierte Sicht, die der Film auf die Bewegung wirft, wird so im Nachhinein beglaubigt, und die gerade durch die Künstlichkeit des Musicals deutlich als fiktionale Erzählung ausgewiesene Handlung wird durch das Schlussbild wieder an die historische Realität zurückgebunden. Weil aber das dokumentarische Schlussbild quasi snatürlich < am Ende des Kamerazooms steht, wird hier nicht nur die Fiktion mit der Realität gedeckt, sondern zugleich auch auf die Narrativität von Geschichte verwiesen.

Nicht nur an die zeitgeschichtliche, sondern auch an die biografische Realität des Autors eng angebunden ist Heinrich Bölls Erzählung Die ver- 
lorene Ehre der Katharina Blum (1974). Das Buch, das die mediale Verfolgung einer unter Terrorismusverdacht geratenden jungen Frau schildert, reflektiert zum einen das angespannte gesellschaftliche und politische Klima der RAF-Jahre. Zum anderen ist es, wie Böll selbst schreibt, eine ganz persönliche Replik, nämlich eine Reaktion auf die Angriffe, die Böll nach seinem Spiegel-Artikel »Will Ulrike Gnade oder freies Geleit?« (1972) erfuhr, in dem er der »Bild«-Zeitung Vorverurteilung und unfaire Berichterstattung vorwirft. In konservativen Kreisen gilt er nun an als Sympathisant, gerät selbst in die Mühlen medialer Kritik. Der Roman ist also auch zu lesen als eine persönliche Stellungnahme. Er sei »ein Pamphlet« gewesen, schreibt Böll zehn Jahr später (1984), »eine Streitschrift«.

Das Ziel des dokumentarischen Gestus ist, das zeigt sich an diesen Beispielen, weniger der Verweis auf Realität als die Behauptung von Realismus. Mit ihm wird nicht nur Bezug auf Realität genommen - wie es fast jedes kulturelle Artefakt tut - , er ist auch nicht einfach Darstellung oder Abbildung von Realität bzw. Kritik an ihr. Der dokumentarische Gestus ist ein formensprachliches Verfahren, das different zu diesen kulturellen Bezugnahmen ist. Er macht aus dem Verweis auf etwas Anderes, nämlich auf das real sich Ereignende, etwas Eigenes, nämlich ein spezifisches ästhetisches Element eines Artefakts. Er zeigt damit eine Differenzqualität in Bezug auf die Realität, einen spezifischen Wirklichkeitscharakter. Darum greift dieses ästhetische Verfahren auch dort, wo der Verweis auf Realität nur noch als formal zu bezeichnen ist, wie etwa, um ein scheinbar fern liegendes Beispiel zu nennen, in den diversen »Reportagen« des Softsexfilms der 1970er Jahre. Der erste Schulmädchenreport, Blaupause für unzählige weitere Schulmädchen-, Krankenschwestern- und Hausfrauenreporte, fußt zwar tatsächlich auf einem 1970 erschienenen Interviewband von Günther Hunold. Doch dieser dient nur als erste Inspiration. Die Filme zeigen keine soziologische Studie zur pubertären Sexualentwicklung, sondern als realistisch behauptete erotische Spielhandlungen und greifen zur Beglaubigung dieses Realismus besonders in den ersten Jahren vielfältig auf klassische film- und fernsehdokumentarische Mittel zurück: Der Moderator Friedrich von Thun macht Straßeninterviews und begrüßt die Kino-Zuschauer aus einem Studio, die Spielszenen werden durch Off-Kommentare unterbrochen und eingeordnet, der Ton in den Moderationen wie in den Off-Texten ist wissenschaftlich-aufklärend. Darin werden die »natürlichen« Handlungen der Teenager gegen die falschen und antiquierten Moralvorstellungen der Eltern verteidigt. Dokumentarische und fiktionale Sequenzen sind miteinander verzahnt und vermischt, das Dokumentarische beglaubigt die Spielszene, die wiederum die (vermeintlichen) Fakten illustriert - ein Verfahren, wie es bis 
heute in zahlreichen Geschichts-Dokumentationen, insbesondere den ZDFHistory-Sendungen regelmäßig eingesetzt wird.

Die bisher genannten Beispiele sind geprägt durch vermeintliche oder echte Bezüge zur Realität. Dokumentarische Mittel lassen sich aber auch finden in einer TV-Serie, die zunächst einmal als ganz und gar fiktionale Narration daherkommt: Der Vorspann der 1970er Jahre Kult-Serie Die Zwei erzählt den bisherigen Lebenslauf der Hauptfiguren Danny Wilde und Brett Sinclair in einer Splitscreen-Collage mit Fotos, Zeitungsausschnitten und vermeintlich »echten« Dokumenten wie den Pässen. Der Zuschauer sieht schon im Vorspann die Vita der Protagonisten, die sie beide als echte Erfolgstypen zeichnet und zugleich mit den gegensätzlichen Lebensgeschichten (britischer alter Adel vs. amerikanischer Selfmade-Öl-Millionär) ausstattet, die zentral für die Serienerzählung sind. Dass im späteren Verlauf des Vorspanns nicht mehr die Namen »Wilde « und »Sinclair «, sondern Curtis und Moore auftauchen, ist nicht nur dem Starstatus der Schauspieler geschuldet, sondern verweist - wie die Serie insgesamt ${ }^{6}$ - medienreflexiv auf die eigene Gemachtheit, dokumentiert gerade die Fiktionalität. Hier liefert der dokumentarische Gestus also keine inhaltlichen Bezugnahmen zur Realität, sondern stützt im Vorspann zunächst die Illusion der Erzählung - die erst später, eingebunden in die Spielhandlung, von den Figuren unterlaufen wird.

\section{Fazit}

Der Bezug zum Gesellschaftlichen, den Brecht mit seinem Gestus-Begriff stark macht, ist bei den hier gezeigten Beispielen unterschiedlich deutlich: Bei Ton Steine Scherben, Christiane F. oder Hair wird direkt auf geschichtliche Ereignisse verwiesen, die mit dem Artefakt wiederum selbst neu diskursiviert werden. Bei Konstantin Wecker oder dem Schulmädchenreport wird dagegen das »Als-ob« bzw. die Frage nach dem Echtheitsgehalt der Erzählung mit dem Gestus des Dokumentarischen als quasi-real beantwortet, während im Fall von Die Zwei das Dokumentarische als ästhetische, narrative Strategie auftaucht, die nur insoweit auf soziale Realität verweist, als diese Art von Zeitungsbildern bekannt ist - wobei das Leben derer, über die in den zu den Bildern gehörenden Artikeln berichtet wird, selbst wieder

6 Die Serie spielt häufig mit einem Durchbrechen der so genannten »vierten Wand « - es wird etwa auf das Vorhandensein von Zuschauern und auf Quoten rekurriert, ironisch mit Genrekonventionen gespielt oder die Rollenbiografie und das private Leben der Stars thematisiert. Dies gilt in besonderem Maße für die deutsch synchronisierte Version - aber mit Abstrichen auch für die Originalfassung. 
als eine Art von Erzählung erscheint. Insofern verweist der dokumentarische Gestus selbst hier nicht mehr direkt, sondern mittelbar auf das Gesellschaftliche: als »kritische Demonstration der Geste«, als »Einschreibung dieser Geste, welcher Zeit sie auch angehören mag, in einen Text, dessen sozialer Hintergrund sichtbar ist « (Barthes 1990: 100). Der dokumentarische Gestus bringt so gerade das Fiktionale und das Nicht-Fiktionale zusammen, indem er Fiktionales und Dokumentarisches in ein oszillierendes Verhältnis zueinander setzt. Damit wird die Erzählung des Artefakts beglaubigt und andererseits die Geschichte als erzählbare und erzählte kenntlich gemacht.

Mit dem dokumentarischen Gestus ist ein Verfahren beschrieben, das in den Artefakten der Epoche mit einer gewissen Häufigkeit zu finden ist. Es lassen sich auch aus der Kunst und der Literatur noch weitere Beispiele anführen, etwa der Schriftsteller Rolf Dieter Brinkmann, der - dem Song »Maschinenland « durchaus verwandt - eine Perspektive wie durch eine Kamera einnimmt, Texte aus Aufzählungen montiert, Collagen und Materialbände veröffentlicht. Oder die Performance Art, wenn etwa die Künstler Verletzungen nicht mehr malen, zeichnen oder filmisch behaupten, sondern sich live - real - zufügen. Die Funktionen des dokumentarischen Gestus sind dabei durchaus unterschiedlich. In den meisten Fällen besitzt er eine mehr oder weniger ausgeprägt Beglaubigungsfunktion: Das Dokumentarische zeigt sich dabei nicht als Authentizität, sondern als Darstellungsstrategie, die mit bestimmten ästhetischen Mitteln auf das Prinzip der Dokumentation und auf die Qualität des Dokuments verweist und so Glaubwürdigkeit herstellt. Hier greift der spezifische Wirklichkeitscharakter, den der dokumentarische Gestus herstellt: Das Dokument ist immer als etwas Wichtiges erkennbar, und es bleibt sogar dann als Dokument existent, wenn es gefaked ist. Hierin liegt der Unterschied zu anderen Beglaubigungsstrategien, die sich nicht auf etwas Drittes berufen können, um Überzeugungskraft zu gewinnen. Mit der buchstäblichen oder auch nur ästhetischen Berufung auf ein Dokument als etwas Drittem, Externem wird das Erzählte als wahr und/oder unmittelbar ausgewiesen, ohne wahr sein zu müssen. Dabei kann - muss aber nicht - der Anschein von Neutralität hergestellt werden. Die scheinbare Objektivität kann sogar - wie im Fall von »Maschinenland « oder Rolf-Dieter Brinkmann - gerade das Transportmittel für eine höchst subjektive Perspektive sein. Bei Weckers »Willy« wiederum beglaubigt der dokumentarische Gestus nicht nur die Erzählung, sondern auch die persönliche Nähe des Liedermachers zum Ereignis und ermöglicht damit auf andere Art die subjektive Teilhabe. Außerdem kann das Dokumentarische konkrete Realitätserfahrungen abbilden wie bei Bölls Die verlorene Ehre der Katharina Blum oder bei Ton Steine Scherben, die ein historisches Ereignis zum 
Song machen und sich selbst dadurch zum historischen Ereignis. In beiden Fällen kommen zur Abbildungsfunktion zwei weitere Momente hinzu: Das erste ist Selbstvergewisserung - mit der Veröffentlichung der eigenen Sicht auf die Dinge wird diese Sicht auch fixiert und in den Diskurs um Bedeutung eingebracht. Die Razzia im Rauch-Haus wird nicht nur einfach aufgeschrieben, sondern zu einem wesentlichen Teil der eigenen Milieugeschichte und damit in ihrer ideologischen Einordnung verstetigt. Das zweite ist je nach Blickwinkel ein Moment der Aufklärung oder der Instrumentalisierung - dieser Effekt ist aus dem Bereich des Dokumentarfilms bekannt.

Die Auswahl der hier vorgestellten Gegenstände, die durch unterschiedliche Künste, Genres und ideologische Verortungen zunächst unorganisiert, wenn nicht sogar beliebig zu sein scheint, ${ }^{7}$ erweist sich methodisch als Möglichkeit, diese Facette der Kultur der 1970er Jahre zu extrahieren. Wenn Kultur als »Ensemble von Texten, die ihrerseits wieder Ensembles sind « (Geertz 1983: 259) bzw. als ein Gewebe von Diskursfäden begriffen wird, dann ist die Auswahl nicht nur zu rechtfertigen, sondern notwendig (vgl. auch Baßler 1995). Erst eine Auswahl ermöglicht die Sicht auf die Schnittbilder der Kultur, in denen die intertextuellen Bezüge, die formalen Gemeinsamkeiten (und Unterschiede) sichtbar werden. Zugleich ermöglicht eine einmal gefundene Spur wie die des Dokumentarischen das gezielte Suchen verwandter Texte und kann, wenn diese zu finden sind, die Auswahl und Ordnung ästhetisch begründen, die nötig sind, um Kultur und Geschichte überhaupt beschreibbar zu machen. Fragt man, warum nun gerade der dokumentarische Gestus in den 1970er Jahren so häufig und in so unterschiedlichen Artefakten auftaucht, ${ }^{8}$ spielen seine Beglaubigungs-, Abbildungs- und Vergewisserungsfunktionen eine wichtige Rolle, denn sie bilden Diskursfäden, die die einzelnen Artefakte miteinander und mit der Epoche, aus der sie stammen, verbinden. Weil der dokumentarische Gestus als eine Geste der Beglaubigung, aber auch ein Moment des Festhaltens und der Versicherung begriffen werden kann, verweist er und verweisen durch ihn die jeweiligen Artefakte nicht mehr nur implizit auf den historischen und sozialen Kontext - wie es in einer Auffassung von Kultur als Gewebe von Diskursfäden alle Texte tun -, sondern er tut dies prinzipiell und explizit. Der »untrennbare « Zusammenhang von historischen und formalen Belangen,

7 Dem Einwand des Beliebigen ist mit Geertz (1983) zu begegnen. Nach ihm »kann man mit der Lektüre des Formenrepertoires einer Kultur überall beginnen und an beliebiger Stelle aufhören« (ebd.: 259).

8 »Warum finden Leser zu einer bestimmten Zeit und an einem bestimmten Ort dieses Werk wohl überzeugend? (Greenblatt 1995: 50) - bei den hier versammelten Gegenständen handelt es sich um zumindest in ihrem Segment erfolgreiche und/oder bedeutende Artefakte der Epoche. 
vom dem die Kulturpoetik ausgeht (Montrose 1995: 64f.), wird hier geradezu gegenständlich sichtbar. Das kulturelle historische Umfeld, in dem dieser dokumentarische Gestus eingesetzt und wirksam wird, ist eines des Umbruchs. Die 1970er Jahre können als transitorische Epoche beschrieben werden: Von der Wiederaufbau- und Wachstumsgesellschaft zu Rebellion und von Resopal-Tischen zur RAF, von der Moderne zur Postmoderne. Das Festhalten und Fixieren, Vergewissern und Beglaubigen im dokumentarischen Gestus kann dann begriffen werden als eine ästhetische Referenz auf eine Zeit fundamentaler Umbrüche.

\section{Literatur}

Anon. (1973). »Berlin-Förderung. So exzessiv und schamlos. «In: Der Spiegel, Nr. 22, S. 38-44; online unter: http://www.spiegel.de/spiegel/print/d-41986676.html (Stand vom 23.7.2012).

Anon. (1977). "SOS für SO 36.«In: Der Spiegel, Nr. 13, S. 216-223; online unter: http://www.spiegel.de/spiegel/print/d-40941796.html (Stand vom 30.1.2012).

Barthes, Roland (1990). »Diderot, Brecht, Eisenstein.«In: Ders., Der entgegenkommende und der stumpfe Sinn. Kritische Essays III. Frankfurt/M.: Suhrkamp, S. 94-102.

Berg, Jan (1987). » Der Beute-Gestus . Dokumentarische Exotik im Film.« In: Die andere Welt. Studien zum Exotismus. Hg. v. Thomas Koeber und Gerhart Pickerodt. Frankfurt: Athenäum, S. 345-362.

Berndt, Klaus (1972). »Bastelten Bethanien-Besetzer die Bomben?« In: B.Z. vom 20. April, S. 4.

Böll, Heinrich (1972). »Will Ulrike Gnade oder freies Geleit?« in: Der Spiegel, Nr. 3, S. 54-57; online unter: http://www.spiegel.de/spiegel/print/d-43019376.html (Stand vom 30.1.2012).

Böll, Heinrich (1974). Die verlorene Ehre der Katharina Blum. Köln: Kiepenheuer \& Witsch.

Böll, Heinrich (1984). »10 Jahre später. Nachwort zur Neuausgabe.«In: Die verlorene Ehre der Katharina Blum. Köln: Kiepenheuer \& Witsch.

Brecht, Bertolt (1963). »Neue Technik der Schauspielkunst.«In: Ders., Schriften zum Theater 3. 1933-1947. Frankfurt/M.: Suhrkamp, S. 151-217.

Burde, Wolfgang (1980). "Gestus. Aspekte eines musiksoziologischen Begriffs. «In: Bericht über den Internationalen Musikwissenschaftliche Kongress Berlin 1974. Gesellschaft für Musikforschung. Hg. v. Hellmut Kühn und Peter Nitsche. Kassel: Bärenreiter, S. 530-532.

Christiane F. (1978). Wir Kinder vom Bahnhof Zoo. Nach Tonbandprotokollen aufgeschrieben von Kai Hermann und Horst Rieck. Mit einem Vorwort von Horst Eberhard Richter. Hamburg: Gruner \& Jahr.

Flusser, Vilém (1993). Gesten. Versuch einer Phänomenologie. Bensheim: Bollmann.

Geertz, Clifford (1983). »Deep Play<. Bemerkungen zum balinesischen Hahnenkampf.« In: Ders., Dichte Beschreibung. Beträge zum Verstehen kultureller Systeme. Frankfurt/M.: Suhrkamp, S. 202-260.

Greenblatt, Stephen (1995). "Kultur.«In: New Historicism. Literaturgeschichte als Poetik der Kultur. Hg. v. Moritz Baßler. Frankfurt/M.: Fischer, S. 48-59. 
Hunold, Günther (1970). Schulmädchenreport. Sexprotokolle. München: Kindler.

Jacob, Andreas (2009). »Der Gestus des Improvisatorischen und der Schein der Freiheit. «In: Archiv für Musikwissenschaft 66, H. 1, S. 1-16.

Koch, Gertrud (2010). "Die Gesten des Films, die filmische Geste - gibt es einen Gestus des Films?" In: Gesten. Inszenierung, Aufführung, Praxis. Hg. v. Christoph Wulf und Erika Fischer-Lichte. München: Fink, S. 145-153.

Kuba, Alexander (2005). "Geste/Gestus. "In: Metzler Lexikon Theatertheorie. Hg. v. Erika Fischer-Lichte, Doris Kolesch und Matthias Warstat. Stuttgart: Metzler, S. $129-136$

Montrose, Louis A. (1995). »Die Renaissance behaupten. Poetik und Politik der Kultur.« In: New Historicism. Literaturgeschichte als Poetik der Kultur. Hg. v. Moritz Baßler. Frankfurt/M.: Fischer, S. 60-93.

\section{Diskographie}

Abwärts (1980). »Maschinenland. « Auf: Amok Koma, Zickzack ZZ 10.

Hans-A-Plast (1979). »Rank Xerox. « Auf: Hans-A-Plast, Lava Records TCH 79449.

Mittagspause (1979). »Der lange Weg nach Derendorf.« Auf: Mittagspause. Pure

Freude CK. 1.

Ton Steine Scherben (1972). »Rauch Haus Song. « Auf: Keine Macht für Niemand, David Volksmund Produktion DVP 007.

Konstantin Wecker (1977). »Willy. « Auf: Genug ist nicht genug, Polydor 2371808.

\section{Filmographie}

Christiane F. - Wir Kinder vom Bahnhof Zoo (1981). Regie: Uli Edel.

Die Zwei / The Persuaders (1970/1971). TV-Serie in 24 Folgen. BRD / GB.

Hair (1979). Regie: Milos Forman.

Schulmädchenreport (1970). Regie: Ernst Hofbauer.

\section{Abstract}

This essay is based on the observation that in the 1970s an aesthetic strategy can be found in popular music, as well as in other areas of popular culture, called here >documentary gestus. This gestus is both artistic documentation of real events and artistic orientation alongside reality. It functions as confirmation, (re)presentation, enlightenment and self-assurance, as well as authentication. It can be seen as a reaction to the so-called transitory character of this era.

The paper showcases the documentary gestus by means of individual examples from music, film and literature of the 1970s. It, thereby, introduces a specific strategy of (re)presentation of that time that not merely reproduces reality, but rather makes this very reproduction into some kind of reality itself. 